\title{
The effect of short-term sensory restriction on the tachistoscopic recognition threshold'
}

S. L. MILSTEIN, D. OLESON, AND JOHN P. ZUBEK, DEPARTMENT OF PSYCHOLOGY, UNIVERSITY OF MANITOBA, Winnipeg, Canada

Subjects who were exposed to 5 min of perceptual deprivation (unpatterned light and white noise) or to a similar duration of visual, auditory, or kinesthetic deprivation showed no significant changes in the tachistoscopic recognition threshold for digits relative to two control groups. Similar results were obtained in a second experiment employing a condition of sensory deprivation (darkness and silence).

Two short-duration studies have reported that either a 5(Rosenbaum et al, 1959) or a 50-min period (Friel \& Derogatis, 1965) of sensory restriction, involving a reduction in visual, auditory, and kinesthetic stimulation, can produce a significant lowering of the tachistoscopic recognition threshold for digits and nouns. The purpose of this study is to determine whether a similar facilitatory phenomenon can occur after a 5-min deprivation period of only one modality. Such an effect might be expected since it has been shown that many of the facilitatory changes reported after prolonged durations of multi-modality sensory restriction can result from visual deprivation alone (Zubek, in press).

\section{EXPERIMENT 1}

Method. The sample consisted of 90 male university students randomly divided into six groups, each containing 15 Ss. Two of the groups were control Ss while the remaining four were exposed to different experimental treatments. Each $S$ served in one condition only. Prior to participation, each $S$ was informed that this was an experiment designed to investigate the effect of various forms of relaxation on visual acuity and that all that was required of him was to relax and follow instructions. During the 5-min deprivation period, $S$ sat in a comfortable chair in a room illuminated with an overhead light and decorated with colorful travel posters. The perceptually deprived Ss (PD) were strapped to the chair to restrict movements and wore a white mask, which permitted light but eliminated pattern vision, and earphones producing a white noise somewhat above the threshold of hearing; the visually deprived group (VD) wore a white mask and received normal auditory stimulation; the auditory group (AD) was exposed to white noise and provided with magazines as additional visual stimulation; and finally, the kinesthetic group (KD) was strapped to the chair but received normal visual and auditory stimulation. In addition, two control groups were employed. One group merely sat in the chair with reading material while the other spent the $5 \mathrm{~min}$ in a students' common room.

The visual recognition threshold for each $S$ was determined immediately before and $2 \mathrm{~min}$ after the termination of the 5-min deprivation or control period by presenting, one at a time, eight randomly constructed five-digit numbers, composed of the integers $1,4,6,7$, and 9 in which no number appeared more than once. These five integers, $3 \mathrm{~mm}$ in height, were selected on the basis of a pilot study in which it was shown that they were similar in difficulty of recognition. The 2 -min interval between the end of the deprivation period and the posttest provided for adaptation to nor mal light in the PD and VD groups. A similar delay interval was employed in the Rosenbaum et al (1959) 5-min deprivation study. The ascending method of limits was used. Beginning with a tachistoscopic exposure of $1 \mathrm{msec}$, a different five-digit number was successively presented, at increasing durations of $1 \mathrm{msec}$, until the number was correctly identified. In order to minimize the effects of practice, two lists of numbers were employed, one before and the other after the 5-min experimental period. The order of presentation of the five-digit numbers in each list was randomly determined using a table of random numbers. The measure of visual efficiency employed was the mean of the recognition threshold obtained on each of 10 trials.

Results. Simple one-way analyses of variance, fixed model for independent measures (Winer, 1965), were used to test the significance of the differences between the amounts of change shown by the six groups of Ss. The statistical analyses were made in terms of difference scores obtained by subtracting the post- treatment from the pretreatment test scores for each $\mathrm{S}$. The results revealed that all six groups of Ss showed a lower recognition threshold, and of approximately the same magnitude, on the 10 posttrials relative to the 10 pretrials. Furthermore, this improvement in visual performance was shown by approximately the same number of $S s$ in each condition. An analysis of variance of the pre-post difference scores indicated that none of the changes were significantly different from each other. Similar results appeared when a comparison was made of the first five pre- and posttreatment trials. Finally, an analysis of variance performed to test the interaction between the various treatments and the 10 postexperimental trials was significant $(F=5.24, p<.01)$. In view of this significant interaction, it is not possible to test for a trend over trials. However, since an improvement in performance was observed in all six groups it is obvious that a practice effect is operating.

\section{EXPERIMENT 2}

Since Rosenbaum et al (1959) reported a lowering of the tachistoscopic recognition threshold of five-digit numbers after $5 \mathrm{~min}$ of either perceptual (unpatterned light and white noise) or sensory deprivation (darkness and silence), a second experiment, employing this latter condition, was conducted. Two groups of 14 Ss each were employed. The experimentals, wearing a black mask and earplugs, were strapped in the chair for $5 \mathrm{~min}$ while the controls spent the same duration in the students' common room. The testing procedure was identical to that of the previous experiment.

The results again revealed that both the experimental and control Ss possessed a lower recognition threshold on the posttreatment relative to the pretreatment trials. However, none of the differences between the two groups of Ss were statistically significant, either on all 10 trials or on the first five trials of the pre- and posttest sessions.

\section{DISCUSSION}

The results of these two experiments indicate that a 5-min period of either sensory or perceptual deprivation or deprivation of any one modality does not produce a significant lowering of the tachistoscopic recognition threshold relative to control Ss. Since any effect resulting from a 5 -min deprivation period would presumably be transitory in nature, it is possible that the length of the posttest session, $15 \mathrm{~min}$ in duration, may have masked any facilitatory effect. However, the presence of nonsignificant differences obtained in a comparison of the first five pre- and posttreatment trials, collected in half the time, suggests that a masking effect probably did not occur.

These negative results are contrary to those obtained by Rosenbaum et al (1959) who, in a study also using five-digit numbers, reported "an improvement in visual efficiency following 5 min of deprivation." Surprisingly, no such effect occurred after longer durations of 15 and $30 \mathrm{~min}$. An examination of their experimental procedure, however, suggests two variables that may have led to positive results. One of these is their improper use of a Latin square design. In discussing practice effects, Winer (1965) warns that "if such effects exist, randomizing or counterbalancing does not remove them; rather, such procedures completely entangle the latter with treatment effects." In view of the strong practice effect observed in both the Rosenbaum and present study, the use of a Latin square design by the former probably produced a confounding of results. The second, and perhaps most important variable, is their use of the same five-digit number which leads to partial recognition at each exposure duration, thus introducing a learning factor in the threshold determination. This may be a critical factor in accounting for their facilitatory effect since Jaffee (1966) has reported a significant improvement in learning ability after $5 \mathrm{~min}$ of sensory deprivation. This problem of partial recognition was eliminated in the present study by changing the stimulus after each presentation and by making the numbers so similar that a guess based on partial recognition had a very low probability of being correct.

The inconsistent finding of an improved performance after 5 
but not after the longer periods of 15 and 30 min of sensory and perceptual deprivation make the Rosenbaum results even more suspect. This inconsistency is not adequately explained by their statement that an absence of an increase in visual efficiency after the longer durations is due to "increased boredom and emotional interference accompanying lengthened isolation." If this explanation was valid, no facilitatory effects should occur after prolonged deprivation periods of one to seven days, a finding contrary to the experimental literature (Zubek, in press). Furthermore, a lower tachistoscopic recognition threshold should not have been obtained by Friel \& Derogatis (1965) after 50 min of perceptual deprivation.

Recently, an increasing number of investigators (e.g., Jackson \& Pollard, 1962; Zubek, 1964) have begun to question whether many of the behavioral and physiological changes that have been reported in the short-term deprivation studies are really due to reduced sensory input. Among them is Cameron et al (1961) who concluded that "periods of exposure of less than one day probably do not produce changes properly attributable to reduction in input." The results of this study substantiate this statement and lead to the conclusion that a 5-min deprivation period does not affect the tachistoscopic recognition threshold. Furthermore, in the other short-term studies in which significant findings were reported the effects probably resulted from inadequate experimental procedures or from such $\mathrm{S}$ variables as expectancy and set which are known to affect deprivation results (Jackson \& Pollard, 1962).

\section{REFERENCES}

CAMERON, D. E., LEVY, L., BAN, T., \& RUBENSTEIN, L. Sensory deprivation: Effects upon the functioning human in space systems. In B. E. Flaherty (Ed.), Psychophysiological aspects of space flight. New York: Columbia Univ. Press, 1961. Pp. 225-237.

FRIEL, C. M., \& DEROGATIS, L. The effect of nonpatterned sensory deprivation on visual recognition thresholds. Psychon. Sci., 1965, 3, 163-164.

JACKSON, C. W., Jr., \& POLlaRD, J. C. Sensory deprivation and suggestion: A theoretical approach. Behav. Sci., 1962, 7, 332-342.

JAFFEE, C. L. The effect of short-term sensory deprivation on rote learning. J. Psychol., 1966, 64, 127-133.

ROSENBAUM, G., DOBIE, S. I., \& COHEN, B. D. Visual recognition thresholds following sensory deprivation. Amer. J. Psychol., 1959, 72, 429-433.

WINER, B. J. Statistical principles in experimental design. New York: McGraw-Hill, 1962.

ZUBEK, J. P. Effects of prolonged sensory and perceptual deprivation. Brit. Med. Bull., 1964, 20, 38-42.

ZUBEK, J. P. Sensory and perceptual-motor processes. In J. P. Zubek (Ed.), Sensory deprivation: Fifteen years of research. New York: AppletonCentury-Crofts, in press.

\section{NOTE}

1. This research was supported by the Defence Research Board, Canada (No. 9425-08) and by the National Research Council, Canada (APA-290). 